\title{
OPTICAL EMISSION SPECTROSCOPY OF MAGNETHRON DISCHARGE Ar/Cu PLASMA
}

\author{
A. Murmantsev ${ }^{a, *}$, A. $\operatorname{Veklich}^{a}$, V. Boretskij ${ }^{a}$, A. Shapovalov ${ }^{b}$, \\ A. KALENYUK ${ }^{c}$ \\ ${ }^{a}$ Taras Shevchenko National University of Kyiv, 64/13, Volodymyrska str., Kyiv, Ukraine \\ ${ }^{b}$ V. N. Bakul Institute for Superhard Materials, National Academy of Sciences of Ukraine, Avtozavodska Str., 2, \\ Kyiv 04074, Ukraine \\ ${ }^{c}$ G. V. Kurdyumov Institute for Metal Physics, National Academy of Sciences of Ukraine, Academician \\ Vernadsky Blvd., 36, Kyiv 03142, Ukraine \\ * murmantsev.aleksandr@gmail.com
}

\begin{abstract}
Plasma parameters (excitation temperature and electron density) of pulsing magnetron discharge is studied by optical emission spectroscopy. Such discharges are usually used as effective sources in sputtering or deposition processes. Vapor admixtures in argon plasma define mainly the temperature and electron density in such discharges. This is the feature, which is typically takes place in plasma of discharge between contacts/electrodes in switching devices of electric technology circuits.
\end{abstract}

Keywords: magnetron, argon plasma, spectroscopy, Boltzmann plots.

\section{Introduction}

Magnetron sputtering is a plasma technology in which atoms of inert gas (usually argon) are ionized and accelerated as a result of the potential difference between a negatively biased (with negative potential) cathode and anode. The interaction of accelerated ions with the cathode surface causes sputtering of atoms which condense on the substrate and form a film $[1,2]$. The condensation and growth of films often occur in conditions far from the thermodynamic equilibrium, due to the presence of kinetic constraints $[2,3]$. Thus, the real control over the thermodynamic and kinetic factors of the deposition process provides an opportunity to control the growth of the films and allows to change the structural, optical, electrical and mechanical properties of the films [4]. One way of controlling the growth of a film is to heat the substrate during its deposition [2]. The deposition temperature affects the energy of the adatoms on the substrate surface, from which the condensate layer is formed [2]. This energy is critical for the activation of surface and mass diffusion $[5,6]$ and allows you to control the morphology of the deposited film. Another source of energy is the plasma particles that transfer energy and impulse to adatoms [5, 6] of the deposited film. At magnetron sputtering, the degree of ionization of plasma particles is relatively low [7], in addition, the quantity of ionization of sputtered cathode atoms is usually less than $1 \%$ [7]. As a result, upon the magnetron deposition of films by direct current the plasma particles have relatively low energies.

In the mid-1990s Mozgrin et al. [8] and Bugaev et al. [9] demonstrated that the use of an ordinary magnetron voltage source in pulsed mode (pulse duration from $1 \mu \mathrm{s}$ to $1 \mathrm{~s}$ and frequencies below $1 \mathrm{kHz}$ ) leads to an increase in pulse currents by two orders of magnitude as compared to the average currents in a conventional magnetron working at DC (DC magnetron) $[8,9]$. The use of these high-pulse currents led, in turn, to the formation of a plasma with electron density of about $10^{18} \mathrm{~m}^{-3}[8,9]$, which significantly exceeds for DC magnetrons (the density of $10^{14}-10^{16} \mathrm{~m}^{-3}$ ) [10]

This study is focused on the discharge plasma parameters of magnetron voltage source in pulsed mode for material sputtering. Optical emission spectroscopy (OES) are used to obtain excitation temperature of $\mathrm{Ar}$ and $\mathrm{Cu}$ atoms, as well as, electron density in plasma. Such parameters at the next step of investigation should make it possible to optimise the deposition processes.

\section{Experimental setup}

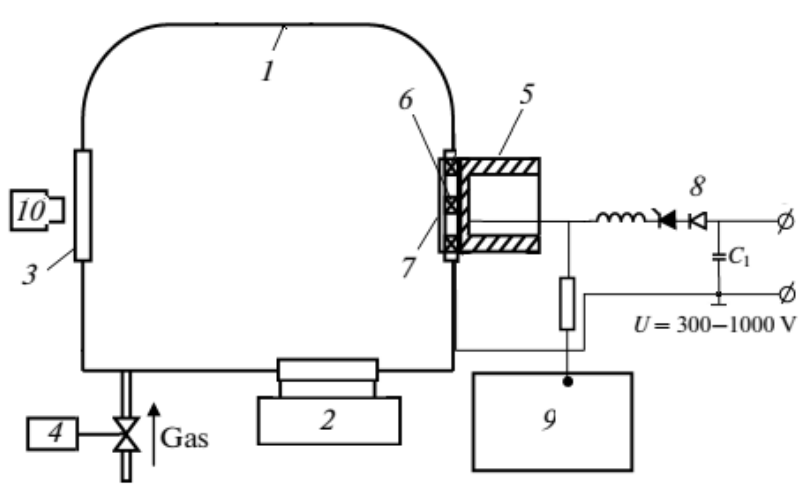

Figure 1. Scheme of the experimental setup.

Investigation are carried out using experimental setup shown in Fig. 1. Discharge was ignited in vacuum chamber (1). Initial vacuum (residual gas pressure of 
$0.4 \mathrm{~Pa}$ ) is created by vacuum pump (2). Discharge parameters and ambient atmosphere were controlled by pulsed power source (8), magnetron (5), ring magnet (6) and gas flow meters (4) respectively. The sputtered target $(7)$ is placed at the vacuum chamber to realise plasma treatment. The discharge power is controlled by two-channels oscilloscope (9). Plasma optical emission is registered through diagnostic windows (3) by Solar LS SDH-IV spectrometer (10) with a 4-position manually switchable diffraction gratings turret. The spectroscopy measurements are carried out at magnetron pulse voltage $550 \mathrm{~V}$ with pulse duration $100 \mathrm{\mu s}$ and are performed in wide spectral range 444-908 nm [11]. Spectral sensitivity of this device is determined by tungsten band-lamp calibration and is taken into account in spectral data treatment (see Fig. 2).

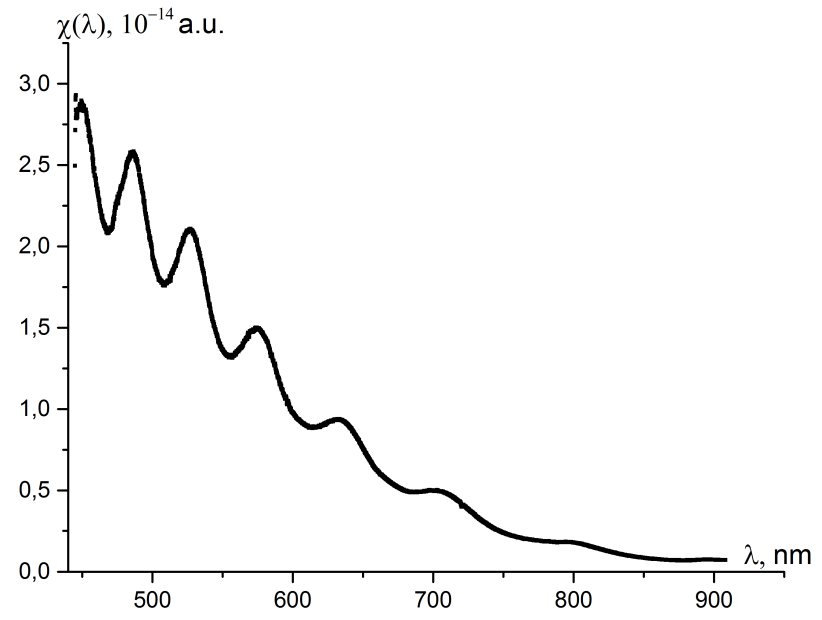

Figure 2. Spectral sensitivity of spectrometer.

\section{Results and Discussions}

It must be noted that spectral lines of different elements are observed during the spectroscopy measurements at the stage of vacuum chamber pumping and at the stage of invariable argon atmosphere pressure of $4 \mathrm{~Pa}$. During the pumping process, just $\mathrm{Cu} \mathrm{I}$ and $\mathrm{H}_{\alpha}$ lines are mainly observed (see Fig. 3). The presence in spectra of such elements is due to the fact that they are presented in chamber volume as desorbed material from construction elements. At the stage of the invariable argon atmosphere $\mathrm{Cu} \mathrm{I}$ are disappeared and Ar I with $\mathrm{H}_{\alpha}$ spectral lines can be clearly registered (see Fig. 4).

Spectral lines profiles are fitted by Gaussian or Voigt function depending on the dominated broadening mechanism. The approximated experimental profiles of $\mathrm{Cu} \mathrm{I}$ and $\mathrm{Ar} \mathrm{I}$ lines are shown in Fig. 5 and 6. As one can see, there is the clearly observed additional noise signal of sinusoidal form in the spectrum. This signal must be withdrawn from consideration in measurement of spectral line intensity. For spectrum with Ar I lines this noise is not taken into account, since its effect was specially minimized and it can be neglected.

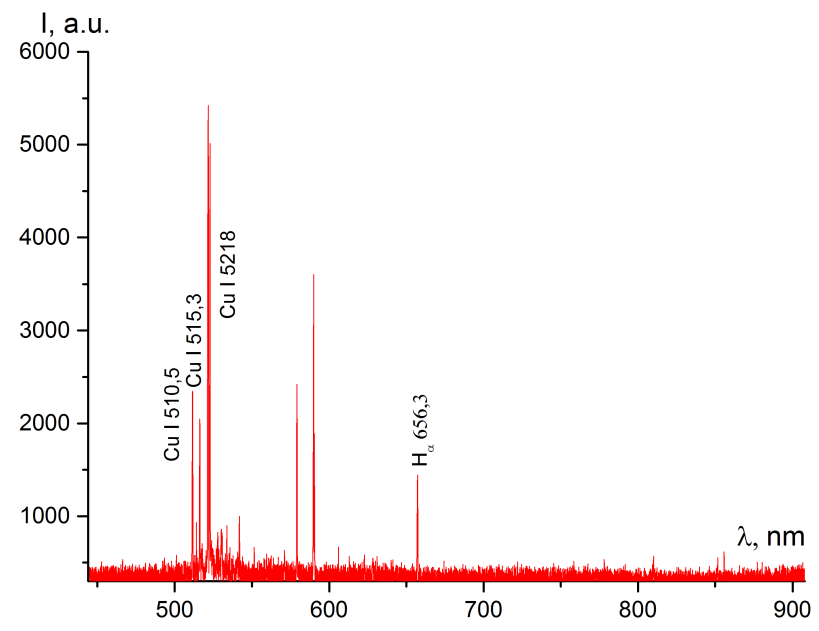

Figure 3. The spectrum with marked $\mathrm{Cu} I$ and $H_{\alpha}$ spectral lines at the stage of vacuum chamber pumping.

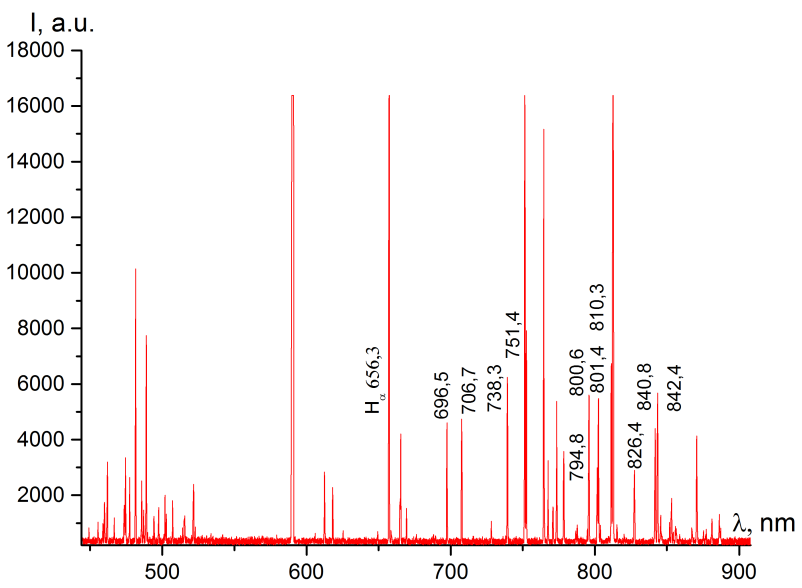

Figure 4. The spectrum with marked Ar I and $H_{\alpha}$ spectral lines at the stage of invariable argon atmosphere.

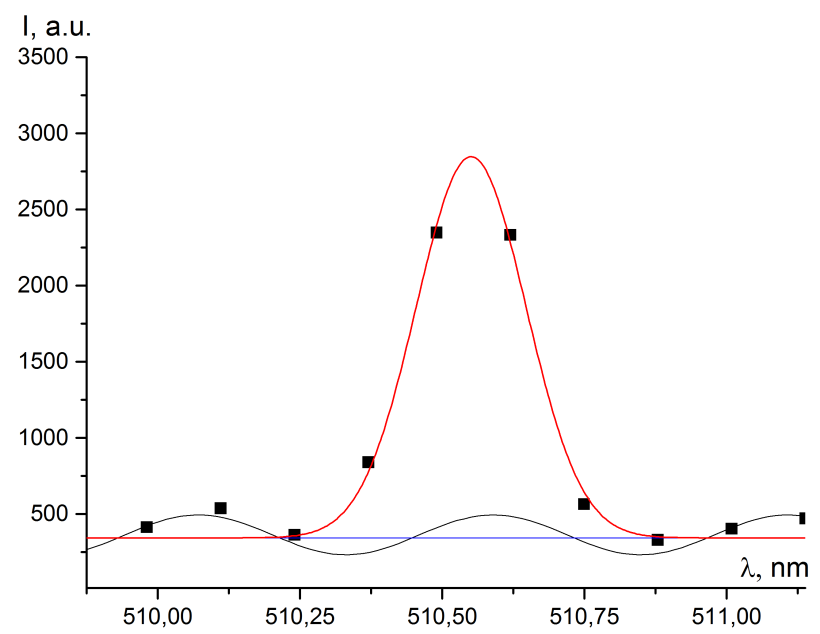

Figure 5. The approximation of Cu I $510.5 \mathrm{~nm}$ spectral line profile by Gaussian. 


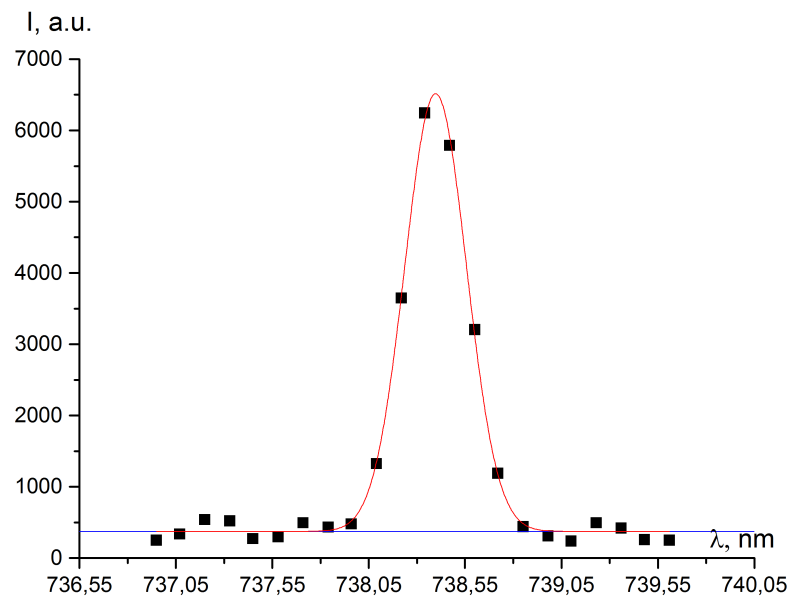

Figure 6. The approximation of Ar I $738.3 \mathrm{~nm}$ spectral line profile by Gaussian.

The electron density in plasma is obtained from half-width of $\mathrm{Cu}$ I $515.3 \mathrm{~nm}$ spectral line broadened by dominated mechanism of quadratic Stark effect [12]. The Lorentz function is typical approximation for this line, but the Voigt function is used in this study. The matter is that the spectrometer instrument function must be taken into account as Gaussian component of Voigt approximation profile (see Fig. 7). Just the Lorentz component of this profile is used to obtain the electron density.

Also for comparison, the electron density was calculated from the half-width of the $\mathrm{H}_{\alpha}$ spectral line [13], which is attended in spectrum (see Fig. 3). The approximation of this line is shown in Fig. 8.

Electron density, obtained from half-width of $\mathrm{Cu} \mathrm{I}$ $515.3 \mathrm{~nm}$ spectral line is equal $4.1 \times 10^{16} \mathrm{~cm}^{-3}$, from $\mathrm{H}_{\alpha}-1.0 \times 10^{16} \mathrm{~cm}^{-3}$.

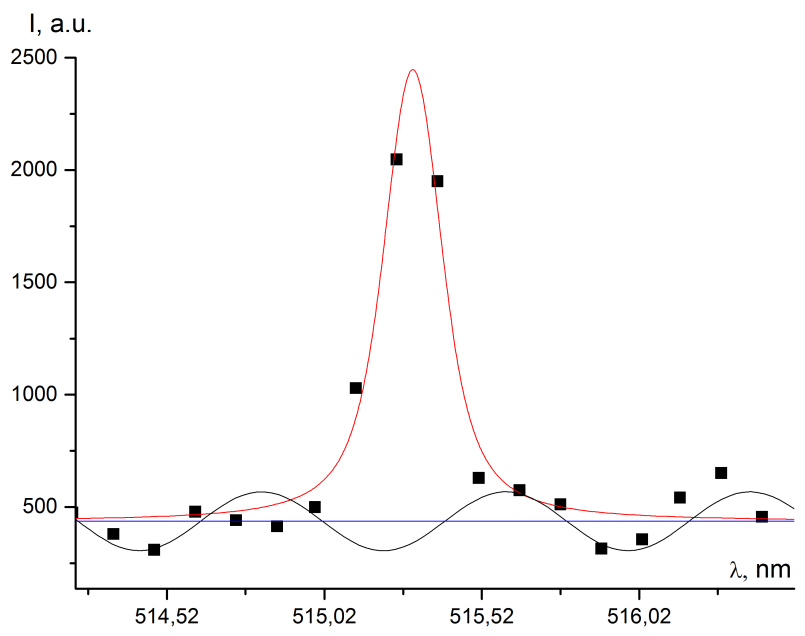

Figure \%. The approximation of Cu I $515.3 \mathrm{~nm}$ spectral line profile for electron density measurements.

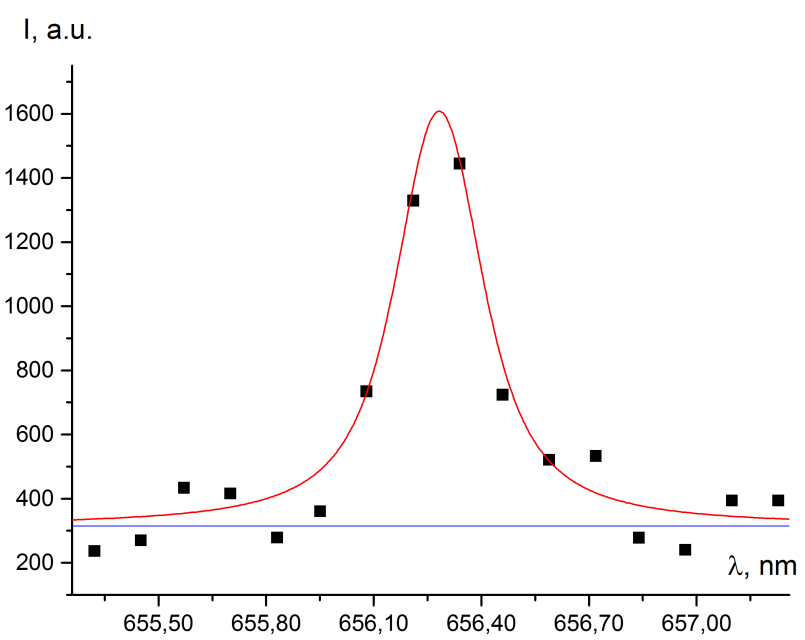

Figure 8. The approximation of $H_{\alpha} 656.3 \mathrm{~nm}$ spectral line profile for electron density measurements.

Plasma excitation temperature is determined by the Boltzmann plot technique. In Fig. 9 and 10 typical Boltzmann plots for $\mathrm{Cu}$ I 510.5, 515.3 and $521.8 \mathrm{~nm}$ and Ar I 696.5, 706.7, 738.3, 751.4, 794.8, 800.6, 801.4, $810.3,826.4,840.8$ and $842.4 \mathrm{~nm}$ are shown. Temperatures are equal $8600 \pm 1200 \mathrm{~K}$, obtained by $\mathrm{Cu}$ I spectral lines and $5400 \pm 1600 \mathrm{~K}$, obtained by Ar I spectral lines, accordingly.

As one can conclude, the determined temperatures are differ considerably. But it must be taken into account few points. Firstly, temperature obtained by Ar I spectral lines is determined with significant error. It can be explained that the registered argon lines have very low difference of the upper energy levels (about $0.3 \mathrm{eV}$ ). This difference should be as much as possible (at least $1 \mathrm{eV}$ ) for optimal application of this technique. Secondly, Cu I and Ar I spectral lines are registered at the different stages of experimental study as it is mentioned above. Thirdly, it is assumed that the plasma is homogeneous during investigation procedure. The last one point must be clarified in the future investigation.

One can turn a special attention that the electron density obtained in this investigation is exceed by more than some orders of magnitude in comparison with $[8,9]$. But it must be stressed that results within frame of this work are determined from two spectral lines of different elements registered simultaneously in the same sample of spectra measurements.

So, the obtained results are presented in Tab.1. 


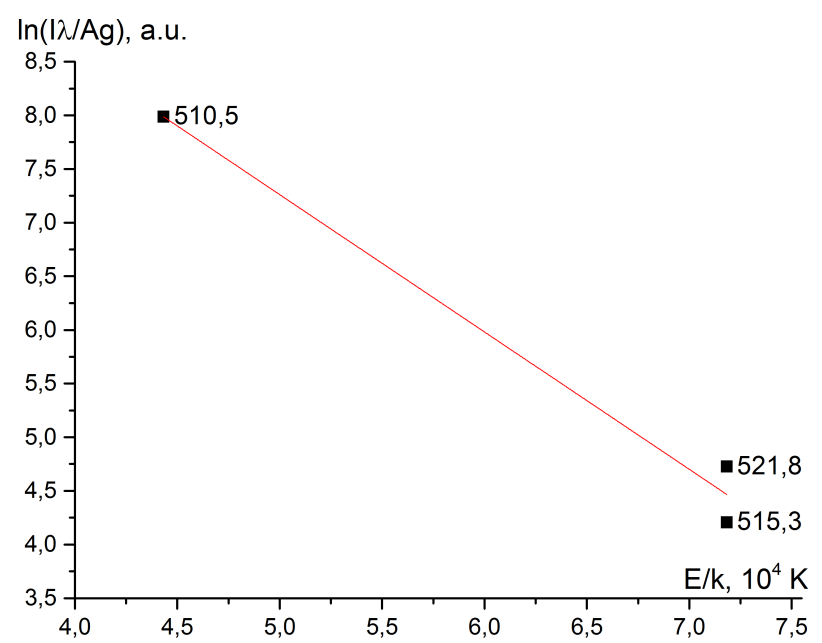

Figure 9. Boltzmann plot on the base of Cu I spectral lines.

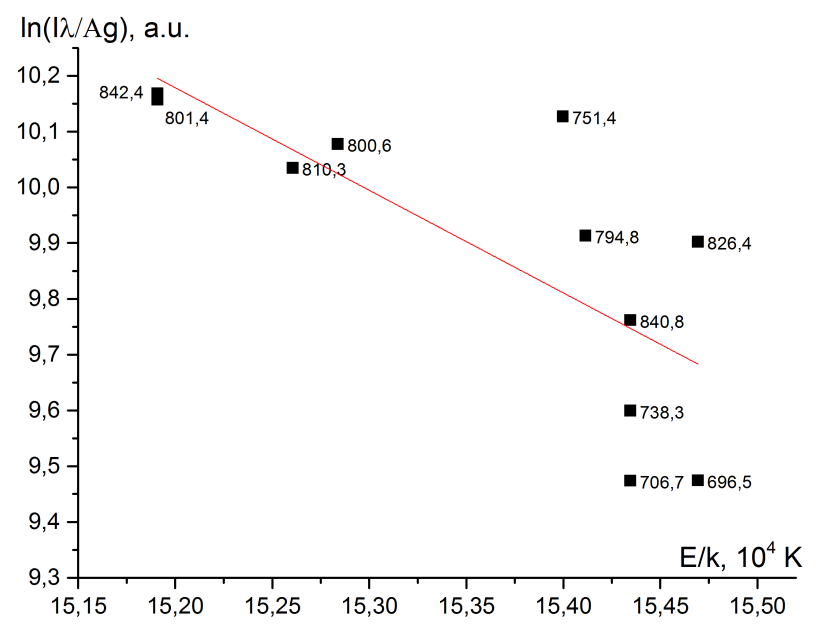

Figure 10. Boltzmann plot on the base of Ar I spectral lines.

\begin{tabular}{ll}
\hline \hline Parameter & Value \\
\hline$T^{C u}$ & $8600 \pm 1200 \mathrm{~K}$ \\
$T^{A r}$ & $5400 \pm 1600 \mathrm{~K}$ \\
$N_{e}^{C u}$ & $4.1 \times 10^{16} \mathrm{~cm}^{-3}$ \\
$N_{e}^{H_{\alpha}}$ & $1.0 \times 10^{16} \mathrm{~cm}^{-3}$ \\
\hline \hline
\end{tabular}

Table 1. $T^{C u}$ - temperature obtained by Cu I spectral lines, $T^{A r}$ - temperature obtained by Ar I spectral lines, $N_{e}^{C u}$ - electron density, obtained from half-width of Cu I $515.3 \mathrm{~nm}$ spectral line, $N_{e}^{H_{\alpha}}$ - electron density, obtained from half-width of $H_{\alpha}$ spectral line.

\section{Conclusions}

Optical emission spectroscopy is suggested to obtain the discharge plasma parameters of magnetron voltage source in pulsed mode.

Excitation temperatures of $\mathrm{Cu}$ and $\mathrm{Ar}$ atomic levels population are determined from Boltzmann plots. The difference of these obtained temperatures can be explained by partial variation of plasma composition at different stages of vacuum chamber operation.

The electron density is determined from half-width of $\mathrm{H}_{\alpha}$ and $\mathrm{Cu} \mathrm{I} 515.3 \mathrm{~nm}$ spectral lines. The obtained value of electron density is original in frame of study of such magnetron discharge plasma and can be as object of the future investigations.

\section{References}

[1] D. Mattox. The Material Science of Thin Films. Academic Press, San Diego, 1992.

[2] M. Ohring. Handbook of Physical Vapor Deposition (PVD) Processing. Noyes Publications, Westwood, 1998.

[3] T. Michely and J. Krug. Islands Mounts and Atoms. Springer, Berlin, 2004.

[4] O. Auciello and R. Kelly. Ion Bombardment Modification of Surfaces: Fundamentals and Applications. Elsevier Science Ltd, Amsterdam, 1984.

[5] P. Petrov, Barna, L. Hultman, and J. Greene. Microstructural evolution during film growth. Journal of Vacuum Science 85 Technology A: Vacuum, Surfaces, and Films, 21(5):S117-S128, 2003. doi:10.1116/1.1601610.

[6] P. Barna and M. Adamik. Fundamental structure forming phenomena of polycrystalline films and the structure zone models. Thin solid films, 317(1-2):27-33, 1998. doi:10.1016/S0040-6090 (97)00503-8.

[7] C. Christou and Z. Barber. Ionization of sputtered material in a planar magnetron discharge. Journal of Vacuum Science \& Technology A: Vacuum, Surfaces, and Films, 18(6):2897-2907, 2000. doi:10.1116/1.1312370.

[8] D. Mozgrin, I. Fetisov, and G. Khodachenko. Pulsed probe technique for determining the plasma parameters of a high-current low-pressure diffuse discharge. Plasma Physics Reports, 25(3):255-260, 1999.

[9] S. Bugaev, N. Koval, N. Sochugov, and A. Zakharov. XVIIth International on Discharges and Electrical Insulation in Vacuum. Noyes Publications, Westwood, 1996.

[10] B. Chapman. Glow Discharge Processes. John Wiley \& Sons, 1981.

[11] A. Veklich, A. Lebid, T. Tmenova, V. Boretskij, Y. Cressault, F. Valensi, K. Lopatko, and Y. Aftandilyants. Plasma assisted generation of microand nanoparticles. Plasma Physics and Technology, 21:28-31, 2017. doi:10.14311/ppt.2017.1.28.

[12] R. Konjevi'c and N. Konjevi'c. Stark broadening and shift of neutral copper spectral lines. Fizika, 18:327-335, 1986.

[13] R. Venger, T. Tmenova, F. Valensi, A. Veklich, Y. Cressault, and V. Boretskij. Detailed investigation of the electric discharge plasma between copper electrodes immersed into water. Atoms, 40(5):1-17, 2017. doi:10.3390/atoms5040040. 